\title{
Molecular characterization of HIV-1 infection in Northwest Spain (2009-2013): investigation of the subtype F outbreak
}

\author{
Dimitrios Paraskevis $^{\mathrm{a}}$, Evangelia Kostaki ${ }^{\mathrm{a}}$, Apostolos Beloukas ${ }^{\mathrm{a}, \mathrm{b}}$, Angelina \\ Cañizares $^{\mathrm{c}}$, Antonio Aguilera ${ }^{\mathrm{d}}$, Javier Rodríguez ${ }^{\mathrm{d}}$, Marta Grandal ${ }^{\mathrm{e}}$, Berta Pernas ${ }^{\mathrm{e}}$, \\ Angeles Castro-Iglesias é Álvaro Mena ${ }^{\mathrm{e}}$, José D. Pedreira ${ }^{\mathrm{e}}$, Eva Poveda $^{\mathrm{e}}$ \\ ${ }^{a}$ National Retrovirus Reference Center, Department of Hygiene, Epidemiology and Medical Statistics, \\ Faculty of Medicine, National and Kapodistrian University of Athens, Athens, Greece \\ ${ }^{b}$ Institute of Infection \& Global Health (IGH), University of Liverpool, Liverpool, UK \\ ${ }^{c}$ Service of Microbiology, INIBIC-Complejo Hospitalario Universitario de A Coruña, A Coruña, Spain \\ ${ }^{d}$ Service of Microbiology, Hospital Conxo-CHUS, and Department of Microbiology, Universidad de \\ Santiago de Compostela, Santiago de Compostela, Spain \\ ${ }^{e}$ Division of Clinical Virology, INIBIC-Complejo Hospitalario Universitario de A Coruña, Universidade da \\ Coruña, A Coruña, Spain
}

\begin{abstract}
Background. HIV-1 subtype B is the predominant one in European regions several, while other subtypes and recombinants are also circulating with high prevalence. A sub-epidemic of subtype $\mathrm{F}$ with specific characteristics and low response to treatment has been recently identified in Galicia. In this study we investigated the characteristics of the HIV-1 subtype F sub-epidemic in A Coruna and Santiago de Compostela in Northwest Spain.

Methods. 420 newly HIV-1 diagnosed patients during 2009-2013 were enrolled in this study. HIV-1 subtyping was carried out using automated subtyping tools and phylogenetic analysis. Molecular epidemiology investigation of subtypes $\mathrm{B}$ and $\mathrm{F}$ was performed by means of phylogenetic analysis using fast maximum likelihood. Phylodynamic analysis was performed using Bayesian method as implemented in BEAST v1.8.

Results. Subtype B found to be the predominant $(61.2 \%$ and $70.4 \%)$ followed by subtype F (25.6\% and $12.0 \%$ ) in both areas (A Coruña and Santiago de Compostela, respectively). The latter found to mainly spread among men having sex with men (MSM). The vast majority of subtype F lineages from both areas clustered monophyletically, while subtype B sequences clustered in several tree branches. The exponential growth of subtype F sub-epidemic dated back in 2008 by means of phylodynamic analysis. Most of new infections during 2009-2013 occurred within the subtype F transmission cluster.

Conclusions. Subtype F circulates at high prevalence in A Coruña and Santiago de Compostela in Northwest Spain, suggesting that the HIV-1 epidemic in this region has distinct characteristics to the rest of Spain. Subtype F has being spreading among MSM and is currently the most actively spreading network. The single cluster spread of this local sub-epidemic might provide an explanation for the distinct characteristics and the low response to antiretroviral treatment.
\end{abstract}

Keywords

HIV-1; Subtype F; Galicia; Spain; Local sub-epidemic; Molecular epidemiology 


\section{Introduction}

Human Immunodeficiency Virus type 1 has up to date infected 78 million people and 39 million have died (UNAIDS, 2013). HIV-1 is classified into four distinct groups: M (major), O (outlier), N (non-M, non-O) and P (recently identified) (Gao et al., 1999; Peeters et al., 1989; Plantier et al., 2009; Van Heuverswyn et al., 2006). Group M is mostly responsible for the AIDS pandemic and is further divided into nine "pure" subtypes (A, B, C, D, F, G, H, J and K), several sub-subtypes (A1, A2, A3, A4, F1, F2), and recombinants. The latter includes an expanding list of at least 66 recombinant forms (CRFs) (Burke, 1997) and multiple unique recombinant forms (URFs) (www.hiv.lanl.gov/content/sequence/HIV/CRFs/CRFs.html). Existing molecular epidemiology studies involving HIV-1 subtyping and identification of recombinants have been conducted for several purposes. These include mapping the geographic distribution of HIV-1 clades; describing local epidemics; identifying potential sources from other geographic regions; conducting molecular surveillance of HIV-1 outbreaks; and applications to Public Health (Abecasis et al., 2013; Hemelaar et al., 2011; Korber et al., 2000; Leitner et al., 1996; Lewis et al., 2008; Paraskevis et al., 2013 ; Rambaut et al., 2004). Moreover, more detailed descriptions of HIV-1 epidemics have been sought using the more sophisticated analyses available through phylodynamic and phylogeographic methods (Korber et al., 2000; Lewis et al., 2008; Paraskevis et al., 2009; Rambaut et al., 2004 ; Volz et al., 2009).

These kind of analyses have been applied to the study of European epidemics at different levels either using pan-European or regional datasets. Based on these previous findings, the HIV-1 epidemic is changing in Europe over time with regards to the prevalence of non-B subtypes and recombinants (Abecasis et al., 2013; Ciccozzi et al., 2012; Miri et al., 2014; Stanojevic et al., 2012; Thomson et al., 2012 ; von Wyl et al., 2011). Although the epidemic originated in Western Europe in the form of subtype B and still remains the predominant one, several countries are experiencing an increasing prevalence of non-B subtypes or CRFs (Austria, Belgium, Cyprus, Greece, Finland, Portugal, Sweden, Switzerland, etc.) and in some cases non-Bs have surpassed subtype B infections (Cyprus, Finland, Greece, Portugal) (Abecasis et al., 2013). There is no unique pattern of distribution of non-Bs across Europe (Abecasis et al., 2013).

Of all European countries, Spain has one of the largest numbers of HIV-1 infected individuals. The large number of HIV-1 infected individuals, combined with a diverse population often characterized by distinct communities based on cultural and linguistic idioms make Spain an important site for molecular epidemiological studies. Previous molecular epidemiology studies have shown that subtype B still remains the predominantly found subtype (approximately $90 \%$ among newly diagnosed individuals between 2002 and 2005); non-B infections are mostly found in immigrants populations, or amongst heterosexuals having contact with people from highly prevalent non-B subtype (McConnell et al., 2008 ; Yebra et al., 2012). However, an increasing trend of subtype F among MSM has recently been identified in Galicia, Northwest of Spain (Thomson et al., 2012). Subtype F has since been detected in Romania in Europe, as well as in Africa and Brazil (Anta et al., 2013; Apetrei et al., 1997; Apetrei et al., 1998; Bandea et al., 1995; Louwagie et al., 1994; Mbisa et al., 2012 ; Mehta et al., 2011).

The aim of the present study is to fully characterize the HIV-1 epidemic in Galicia by means of molecular epidemiology in light of our recent findings for impaired treatment responses of individuals infected with subtype F (Pernas et al., 2014). Specifically, we estimate the prevalence of different HIV-1 clades in Galicia and look in more detail at the patterns of dispersal for the different subtypes investigating potential associations with transmission risk groups, their origins, transmission dynamics, as well as potential infectivity. HIV-1 sequences from newly diagnosed patients during 2009-2013 sampled from two towns in the Northwest of Spain, A Coruña and Santiago de Compostela were used in the analyses. 


\section{Materials and methods}

\subsection{Study population}

The study enrolled 420 newly diagnosed and treatment naïve HIV-1 patients from 2009 to 2013 from two regions in Northwest of Spain, 278 (66.2\%) from A Coruña and 142 (33.8\%) from Santiago de Compostela. Patient demographics (age, sex, and risk behavior) and laboratory (HIV RNA and HIV pol sequences generated using TRUGENE® HIV-1 genotyping assay) parameters were recorded at the time of diagnosis. All study procedures have been conducted in accordance with the local code of ethics.

\subsection{Phylogenetic analyses}

HIV-1 subtypes were identified using the online automated subtyping tool COMET (http://comet.retrovirology.lu/) (Struck et al., 2015). To provide further confirmation of the COMET results, a two-step phylogenetic analysis was carried out. Initially, phylogenetic analysis was performed on two datasets consisting of 278 sequences from A Coruña and 142 sequences from Santiago de Compostela along with 22 reference sequences belonging to 9 pure subtypes (A$\mathrm{D}, \mathrm{F}-\mathrm{H}, \mathrm{J}$ and $\mathrm{K})$. Subsequently, a more extensive phylogenetic analysis was also performed on both datasets along with 216 reference sequences belonging on 9 pure subtypes $(\mathrm{A}-\mathrm{D}, \mathrm{F}-\mathrm{H}, \mathrm{J}$ and $\mathrm{K}$ ) and 49 circulating recombinant forms (CRFs) (CRF01_AE-CRF49_cpx). All the reference sequences used in the analysis were downloaded from the public open access Los Alamos HIV sequence database (http://www.hiv.lanl.gov). In the absence of any unclassified sequences no further analysis was performed including newly characterized CRFs. MEGA v5.2 was used to align sequences along the reference sequences and alignments corrected manually (Hall, 2013). Phylogenetic analysis performed based on fast maximum likelihood method as implemented in FastTree, using the GTR (general time reversible) as nucleotide substitution model with gamma $(\gamma)$ distribution of rate variability among sites with sites parted on 20 categories (Price et al., 2010). Tree's reliability was also calculated through FastTree using the Shimodaira-Hasegawa test. Tree visualization and annotation was performed using FigTree v1.4 program (Drummond et al., 2012).

Additional phylogenetic analysis for the two predominant subtypes (B and F) was performed in order to examine the patterns of their dispersal. This phylogenetic analysis was performed by including sequences sampled from Galicia and different geographic areas on a global scale as references. Specifically, 14.835 subtype B sequences and 1.120 sequences of subtype F available at Los Alamos HIV sequence database (http://www.hiv.lanl.gov/content/index) on the protease $(\mathrm{PR})$ and reverse transcriptase (RT) genomic fragment were used as references. All the available subtype $\mathrm{F}$ sequences on the database where used as references. Subtype B reference sequences were sampled randomly (Supplementary Tables 1 and 2). All reference sequences were sampled worldwide and their subtype was further verified using the online automated subtyping tool COMET. Phylogenetic analysis was performed as previously described.

\subsection{Phylodynamic analysis}

The phylodynamic analysis was performed using the Bayesian method as implemented in BEAST v1.8 on the two subtype datasets separately (Drummond et al., 2012). Because study sequences had been sampled too recently (2009-2013) for the substitution rate to be estimated, new reference sequences were used with known sampling dates. These reference sequences were chosen based on their sampling date and their city/state of origin (or sampling). Sequences with early sampling dates were preferred in order to maximize the sampling window (e.g. 21 years), 
providing the information needed to estimate the molecular clock rate. Specifically, for subtype $\mathrm{F}$ among the subtype F Galician dataset, the 14 subtype F sequences with the oldest sampling date found on the Los Alamos HIV sequence database were used as references. Similarly, among the subtype B Galician dataset, 9 sequences randomly selected among the subtype B sequences with the oldest sampling date available on Los Alamos HIV sequence database were used as references (Supplementary Tables 3 and 4). Analysis was performed using the generalized time reversible (GTR) with gamma $(\gamma)$ heterogeneity model, an uncorrelated lognormal relaxed clock model with TipDates and a Bayesian skyline non-parametric plot demographic model with 10 groups. Noninformative priors were used for the MCMC runs. Each MCMC chain was run for $30 \times 10^{6}$ and $50 \times 10^{6}$ generations and sampled every 3.000 and 5.000 steps, respectively, with the first $20 \%$ discarded as burn-in $\left(6 \times 10^{6}\right.$ and $10 \times 10^{6}$, respectively). MCMC convergence, tMRCA and effective samples sizes (ESS) were checked using the program Tracer v1.5 (Drummond et al., 2012). Each run consensus tree was implied and the maximum clade credibility (MCC) tree was then selected from the posterior tree distribution by the TreeAnnotator program v1.8 (Drummond et al., 2012).

\subsection{Statistical analysis}

Statistical analysis was carried out using Pearson's chi-squared statistical test as implemented in STATA 12 with 6 degrees of freedom and additionally the Fisher's exact test was also carried out to determine any correlation between subtype and transmission risk group in Galicia.

\section{Results}

\subsection{Descriptive characteristics}

A total of 420 newly diagnosed and treatment naïve patients were enrolled, 278 from A Coruña and 142 from Santiago de Compostela. Table 1 shows the main epidemiological characteristics of the study population. Overall, most were males who acquired HIV-infection by sexual transmission $(92.7 \%$ vs. $88.7 \%$, respectively). However, the distribution by risk group varied between the two cities. Santiago de Compostela was characterized by higher proportions of men having sex with men (MSM) (63.4\%) and injecting drug users (IDUs) (10.5\%), compared with relatively higher proportions of heterosexuals $(39.2 \%)$ in A Coruña $(p<0.001)$. Mean HIV-1 RNA and mean CD4 counts at the diagnosis time were similar for both cities (4.93 vs. 4.94 and 409.47 vs. 392.35 , respectively). 
Table 1. Characteristics of the study population.

\begin{tabular}{|c|c|c|c|}
\hline \multirow{2}{*}{ Characteristics } & \multicolumn{2}{|l|}{$N=420$} & \multirow{2}{*}{$P$} \\
\hline & A Coruña $(n=278)$ & Santiago de Compostela $(n=142)$ & \\
\hline Male $(\%)$ & 86.3 & 84.2 & 0.593 \\
\hline Age & $42.08 \pm 10.15$ & $39.34 \pm 11.6$ & 0.143 \\
\hline Routes of HIV transmission (\%) & & & 0.000044 \\
\hline Heterosexual & 39.2 & 26.1 & \\
\hline $\operatorname{MSM}^{\mathrm{a}}$ & 47.0 & 63.4 & \\
\hline IDUs $^{\mathrm{b}}$ & 7.6 & 10.5 & \\
\hline Others & 6.5 & - & \\
\hline Mean HIV-RNA (log copies/ml) at diagnosis time & $4.93 \pm 0.93$ & $4.94 \pm 0.92$ & 0.516 \\
\hline Mean $\mathrm{CD} 4^{+}$at diagnosis time & $410.84 \pm 306.46$ & $392.35 \pm 272.419$ & 0.586 \\
\hline
\end{tabular}

${ }^{\text {a }}$ MSM: men having sex with men.

${ }^{\mathrm{b}}$ IDUs: injecting drug users.

\subsection{Subtyping outcome}

Analysis revealed that subtype B was the predominant one $(61.2 \%$ and $70.4 \%)$, followed by $\mathrm{F}$ (25.6\% and 12\%) in both A Coruña and Santiago de Compostela, respectively (Fig. 1). Subtype F was the second most prevalent clade with sequences referred to sub-subtype F1. Additionally, detected at low prevalence were subtypes A1 (1.8\% and 3.5\%), C (2.5\% and $4.2 \%), \mathrm{G}(0 \%$ and $1.4 \%$ ), CRFs (6.8\% and 2.8\%) and unique recombinant forms (URFs) $(2.2 \%$ and $5.6 \%$ ) at A Coruña and Santiago de Compostela, respectively (Table 2). In total non-B or non-F subtypes corresponded to the $13.3 \%$ for A Coruña and $17.6 \%$ for Santiago de Compostela. Regarding the distribution of transmission risk groups in different subtypes, MSM were more frequently detected among subtype $\mathrm{F}(78.4 \%)$ than B $(47.8 \%)$ in contrast to heterosexuals and IDUs that were more frequently infected with subtype B. Specifically it was found that $17.1 \%$ and $36.3 \%$ of heterosexuals and $1.1 \%$ and $11.9 \%$ of IDUs were infected with subtype F and B, respectively, totally for both cities $(p<0.001)$ (Table 3$)$.

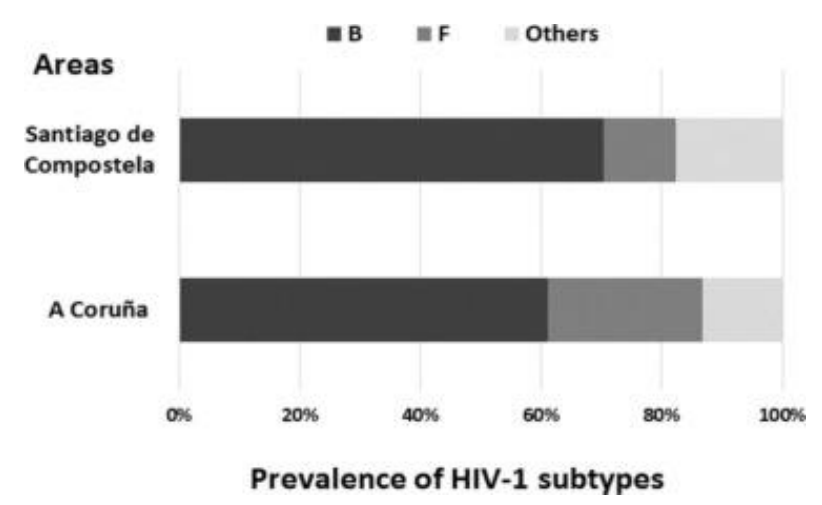

Fig. 1. Prevalence of HIV-1 subtypes in the two Spain cities. 
Table 2. Prevalence of HIV-1 subtypes separately for the two cities.

\begin{tabular}{lll}
\hline & \multicolumn{2}{l}{ Regions } \\
\cline { 2 - 3 } Subtypes & A Coruña $(n, \%)$ & Santiago de Compostela $(n, \%)$ \\
\hline & & \\
B & $170(61.2)$ & $100(70.42)$ \\
F & $71(25.5)$ & $17(12)$ \\
A1 & $5(1.8)$ & $5(3.5)$ \\
C & $7(2.5)$ & $6(4.2)$ \\
G & $0(0)$ & $2(1.4)$ \\
CRFs & $19(6.8)$ & $4(2.8)$ \\
URFs & $6(2.2)$ & $8(5.6)$ \\
Total & $278(100)$ & $142(100)$ \\
& & \\
\hline
\end{tabular}

Table 3. Distribution of HIV-1 transmission risk groups for different subtypes totally for A Coruña and Santiago de Compostela.

\begin{tabular}{llll}
\hline \multicolumn{3}{c}{ HIV-1 subtypes } & \\
\cline { 2 - 4 } & $\mathrm{B}(n, \%)$ & $\mathrm{F}(n, \%)$ & Others, $(n, \%)$ \\
\hline & & & \\
Risk groups & & & \\
MSM $^{\mathrm{a}}$ & $129(47.78)$ & $69(78.41)$ & $22(35.48)$ \\
Heterosexuals & $98(36.30)$ & $15(17.05)$ & $33(53.23)$ \\
IDUs & $32(11.85)$ & $1(1.14)$ & $3(4.84)$ \\
Unknown & $11(4.07)$ & $3(3.41)$ & $4(6.45)$ \\
Total & $270(100)$ & $88(100)$ & $62(100)$ \\
& & & \\
\hline
\end{tabular}

${ }^{\text {a }}$ MSM: men having sex with men.

b IDUs: injecting drug users.

\subsection{Molecular characterization of Northwest Spain HIV-1 epidemic}

Extensive phylogenetic analysis was performed in order to characterize the spread of the epidemic for the two (B and F) major subtypes in Galicia. This kind of analysis can provide evidence about the levels of regional transmission. A single monophyletic cluster for most of subtype $\mathrm{F}$ lineages for both regions was revealed, suggesting a local transmission network, while on the other hand subtype B sequences clustered at different points in the tree (Fig. 2). Subtype B dispersal across the tree indicated multiple introductions from different geographic regions. In a more detailed examination of subtype B either non-clustered sequences or small regional clusters were identified. In some cases these clusters consisted only of sequences from a single region. No specific geographic area was identified as the origin of subtype B in Galicia. The origin of subtype F on the other hand, was probably from Brazil since the Galician cluster appeared as nested within sequences from Latin America (data not shown; Fig. 3). 


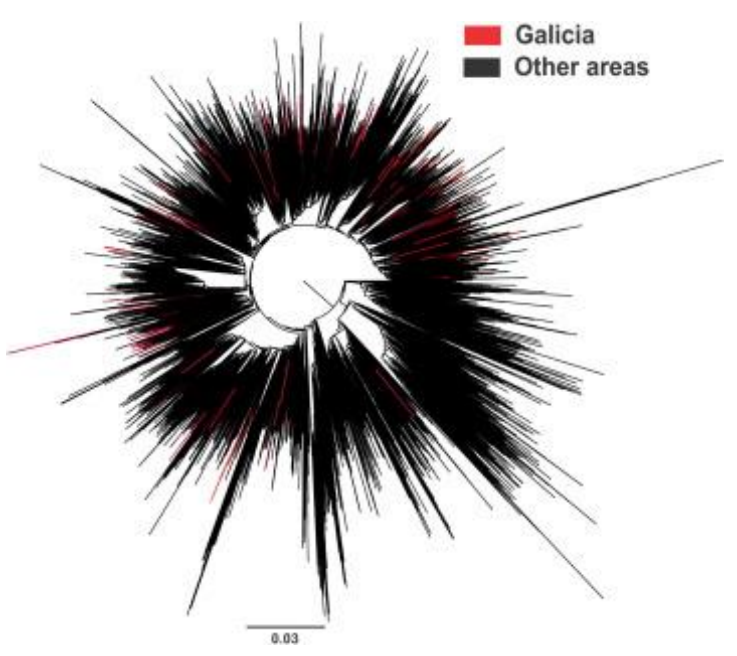

Fig. 2. Phylogenetic analysis of HIV-1 subtype B sequences from Galicia and a global reference dataset. Galician sequences are marked in red in contrast with sequences from other areas marked in black. (For interpretation of the references to color in this figure legend, the reader is referred to the web version of this article.)

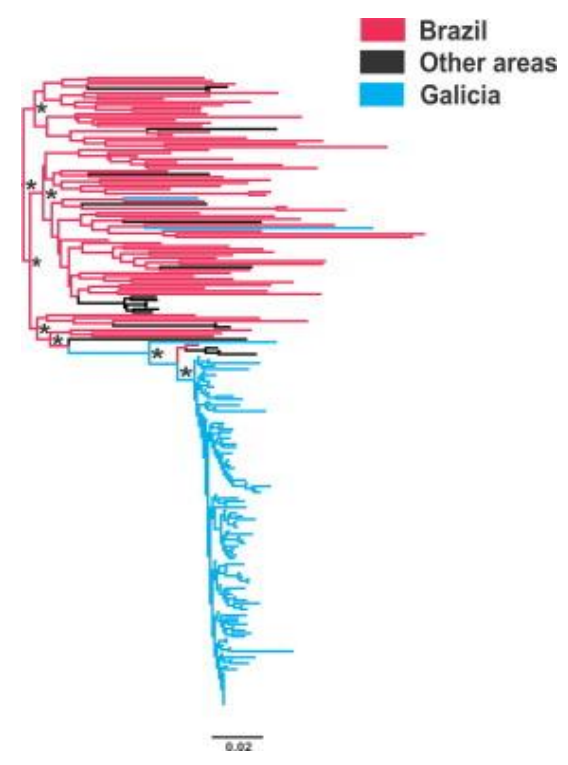

Fig. 3. Phylogenetic analysis of HIV-1 subtype F sequences from Galicia and a global reference dataset. Galician sequences are marked in cyan in contrast with sequences from Brazil and other countries marked in neon red and black respectively. Stars are placed at the internal side of the corresponding tree nodes indicating posterior probabilities higher than 0.75 . (For interpretation of the references to color in this figure legend, the reader is referred to the web version of this article.)

To describe the temporal patterns of the subtype $\mathrm{F}$ regional epidemic, we carried out phylodynamic analysis. Molecular clock analysis revealed that the time of the most recent common ancestor (tMRCA) for subtype F in Galicia was in 1993 (median estimate, 95\% higher posterior density HPD: 1979-2003) (Fig. 4). The tMRCA should be considered as the approximate time of infection of the potential founder of the subtype F epidemic in Galicia sampled in our data. The skyline plot revealed the dynamics of subtype $\mathrm{F}$ in Galicia and showed an unprecedented upward shift in the number of infections during the last five years (Fig. 5). The phylodynamic analyses revealed evolutionary rate equal to $1.791 \mathrm{E}-3$ substitutions/site/year for the Galician subtype F dataset and 2.279E-3 substitutions/site/year for the Galician subtype B dataset, respectively. 


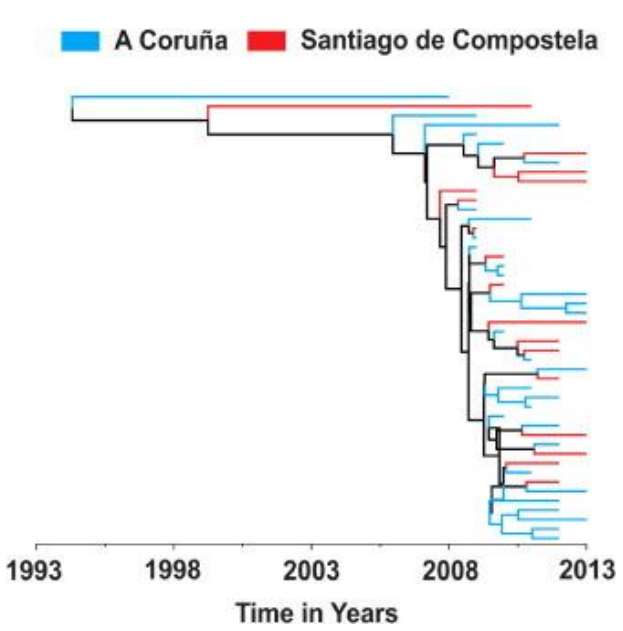

Fig. 4. Dated phylogenetic tree from molecular clock analysis of HIV-1 subtype F sequences from Galicia. Sequences shown in red and cyan correspond to Santiago de Compostela and A Coruña respectively. The Galician sequences analyzed identified local transmission network especially the last 5 years. (For interpretation of the references to color in this figure legend, the reader is referred to the web version of this article.)

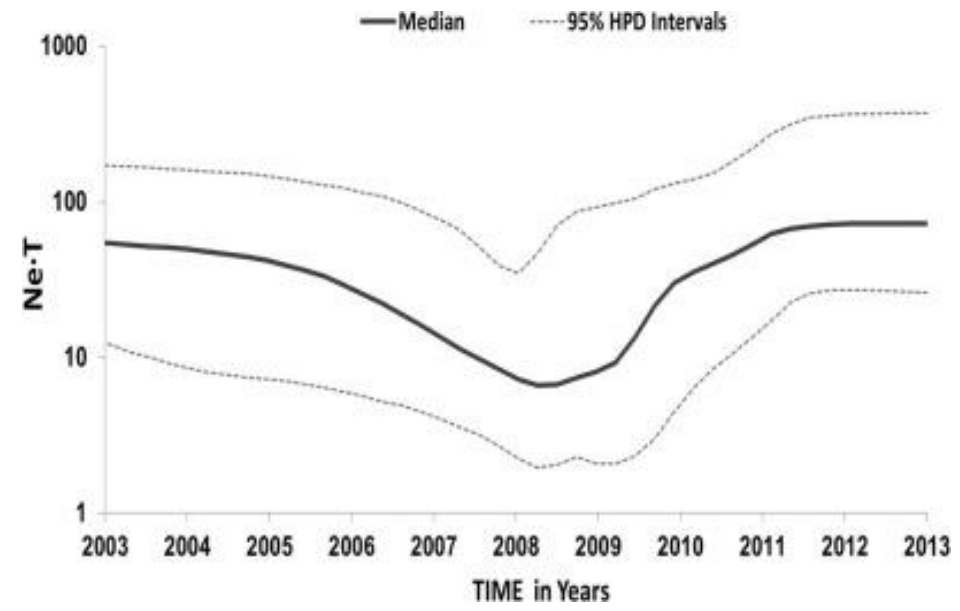

Fig. 5. Skyline plot for subtype F epidemic (median and 95\% HPD intervals estimates) in Galicia showing the effective number of infections $\times$ generation time $(\mathrm{Ne} \cdot \mathrm{T})$ ". 
Based on the inferred dated phylogenies and phylodynamic analysis for subtype $\mathrm{B}$ and $\mathrm{F}$ in Galicia, more of the recent infections (occurred during the last 5 years) corresponded to the coalescent dates of lineages observed for subtype F ( $81 \%$ of all subtype F infections) than subtype B (14\% of all subtype B infections) (Fig. 5 and unpublished data). Regarding the origin of subtype $\mathrm{F}$ a single sequence from Santiago de Compostela was placed as the outlier in the dated tree, however lineages from A Coruña were also highly divergent suggesting that subtype $\mathrm{F}$ was introduced at a similar time in both cities (Fig. 4). Moreover, there was evidence within the tree for monophyletic clustering suggesting local transmission in either cities and across the two areas.

\section{Discussion}

Our findings revealed that both HIV-1 subtypes F and B are highly prevalent in A Coruña and Santiago de Compostela in Northwest Spain. However, each subtype has different characteristics in terms of the pattern of spread epidemic, proportion of transmission risk groups, and origin. As suggested by phylodynamic analysis, infections occurring within the subtype F cluster belong to the only actively propagating transmission network that includes mainly MSM. Crucially as suggested by our recent study subtype $\mathrm{F}$ infected individuals show an impaired response to treatment (Pernas et al., 2014).

Given the particular local spread of a non-B subtype in Galicia initially described by Thomson et al. (2012), we looked into more detail at the characteristics of the HIV-1 epidemic in Galicia by means of molecular epidemiological analyses. We used a large collection of samples from two major cities and by using current state of the art methods for phylogeographic and phylodynamic analyses we estimated the origin and the transmission dynamic for subtype F. Given the rarity of subtype $\mathrm{F}$ in Europe, where Romania is the only endemic area, the monophyletic clustering of subtype F (Apetrei et al., 1997; Apetrei et al., 1998; Bandea et al., 1995; Mbisa et al., 2012 ; Mehta et al., 2011), which is indicative of local transmission, is not surprising. This is can be explained due to the low frequency of this subtype in western countries, from where we expect that the local population in Galicia retains most contacts. Subtype B, on the other hand, was the result of multiple introductions similar to most countries in W. Europe. Looking into the distribution of different subtypes in Europe, Galicia becomes the only area outside of Romania where this clade is circulating at a high rate. Interestingly, the putative origin of subtype $\mathrm{F}$ has been identified in Latin America and Brazil where Spain has historical links. This pattern is unique across Spain where subtype B has been detected at a high prevalence and additional non-B and CRF02_AG have been introduced mainly as a result of immigration from highly prevalent non-B locations.

Galicia, therefore, should be considered as an area with particular characteristics in HIV-1 epidemic with regard to considerable levels of local spread and the high prevalence of subtype F. Strong cultural ties and lingual idiomatic differences of Galicians in comparison with the rest of Spanish populations can explain these unique characteristics. It would be interesting to note whether subtype F spreads into other areas of Spain in the future.

Regarding factors associated with local spread, our findings indicate that MSM constitutes the major risk factor for subtype $\mathrm{F}$ transmission. Molecular clock analysis indicated that subtype $\mathrm{F}$ was introduced approximately 20 years ago in Galicia, however it grew to epidemic proportions only recently. Introduction of subtype F within an active network of MSM probably triggered the subtype F sub-epidemic growth last five years. High-risk behavior of this population was the most probable reason for the exponential growth of subtype $\mathrm{F}$ epidemic. The rapid expansion of this clade among MSM in this region of Spain is alarming and needs action to be taken in order to avoid an extended spread of HIV-1 virus in Galicia. Crucially local stakeholders and policy makers should focus their response to subtype $\mathrm{F}$ infected individuals and their network since as shown clearly in our study; this presents the only actively propagating one during the last years. 
Of note, very recent data has shown that in addition to differences in the transmission route for subtype F, virologic response rates to antiretroviral therapy (ART) were significantly lower among subtype F infected individuals compared to subtype B ones at weeks 24, 48, and 96 weeks after ART initiation. Interestingly, subtype F was independently associated with virological response at 24 weeks. Given the relevant implications for the clinical management of these patients additional studies are warranted to identity reasons for poor ART virologic response in subtype F patients (Anta et al., 2013 ; Pernas et al., 2014).

Our results highlight the benefits of using detailed molecular epidemiological methods for studying epidemics. Monitoring the prevalence of different clades is useful and can provide information about the genetic diversity of circulating strains as well clues about the potential origin of infections. However phylogeographic and phylodynamic analyses are more informative as shown by our study, providing detailed information about the characteristics of the epidemic that can be important for public health. Thus, phylodynamic analysis revealed the transmission dynamics over time for the $\mathrm{F}$ clade which is of crucial importance for our understanding the potential of epidemic growth of a strain circulating among the HIV-1 seropositives; this in turn can be valuable for prevention and thus for Public Health. Moreover phylogeographic analyses can reveal the origin of infections in more detail, which can be of relevance in the prevention of cases within specific populations or human practices.

\section{Conclusions}

The rapid expansion of HIV-1 subtype F local sub-epidemic suggests the existence of transmission networks among MSM who are unaware of their HIV status engaging in high-risk behavior in Galicia. This situation should be treated as one of the highest public health priorities in Spain considering the country's special characteristics, demographic changes taking place worldwide and the fact that HIV-1 is one of the most extended and alarming epidemics globally the last decades.

\section{Acknowledgements}

The study has been supported in part by the Hellenic Scientific Society for the study of AIDS and STDs, also in part by grants from Fondo de Investigación Sanitaria (CP08 / 00214, PI10 / 02166 , PI13 / 02266, CM13 / 00328), and Fundación Profesor Novoa Santos, A Coruña. We would like to thank Biobank of A Coruña (SERGAS) for providing us the technical, ethical and legal advice necessary for the development of our research. 


\section{Appendix A. Supplementary data}

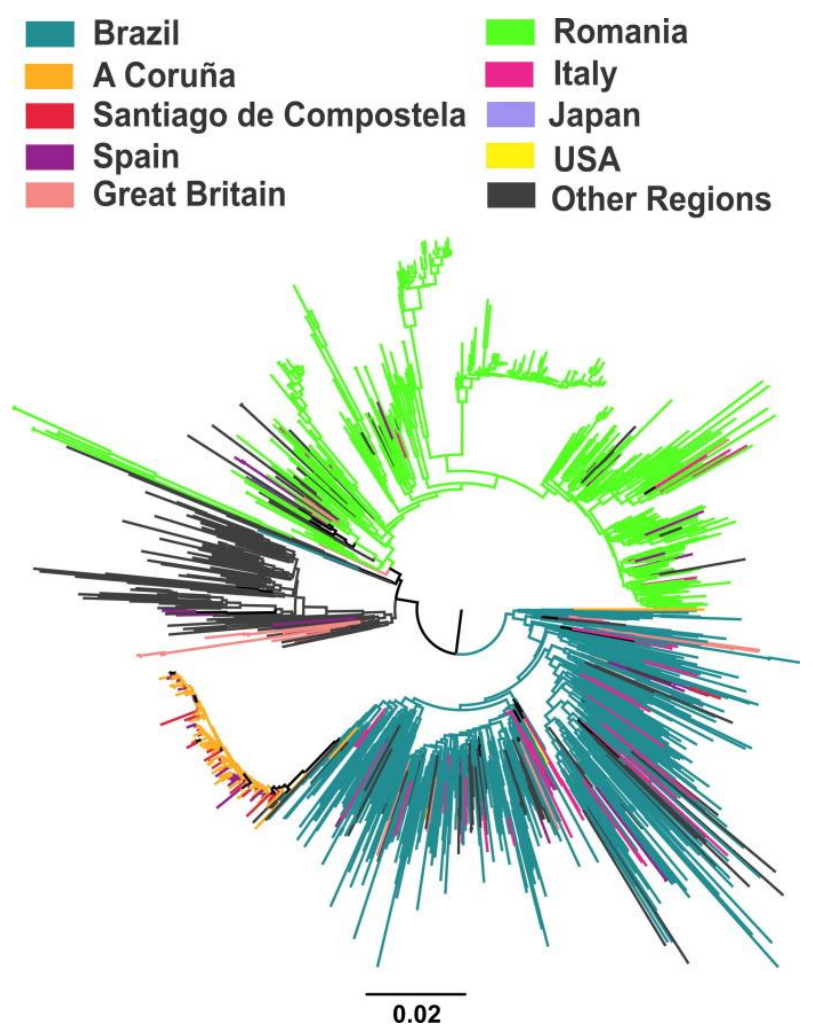

Supplementary figure.

\section{References}

Abecasis, A.B., Wensing, A.M., Paraskevis, D., Vercauteren, J., Theys, K., Van de Vijver, D.A., Albert, J., Asjo, B., Balotta, C., Beshkov, D., Camacho, R.J., Clotet, B., De Gascun, C., Griskevicius, A., Grossman, Z., Hamouda, O., Horban, A., Kolupajeva, T., Korn, K., Kostrikis, L.G., Kucherer, C., Liitsola, K., Linka, M., Nielsen, C., Otelea, D., Paredes, R., Poljak, M., Puchhammer-Stockl, E., Schmit, J.C., Sonnerborg, A., Stanekova, D., Stanojevic, M., Struck, D., Boucher, C.A., Vandamme, A.M., 2013. HIV-1 subtype distribution and its demographic determinants in newly diagnosed patients in Europe suggest highly compartmentalized epidemics. Retrovirology 10, 7

Anta, L., Blanco, J.L., Llibre, J.M., Garcia, F., Perez-Elias, M.J., Aguilera, A., Perez- Romero, P., Caballero, E., Vidal, C., Canizares, A., Gutierrez, F., Dalmau, D., Iribarren, J.A., Soriano, V., de Mendoza, C., Drug Resistance Platform of the Spanish, A.R.N., 2013. Resistance to the most recent protease and nonnucleoside reverse transcriptase inhibitors across HIV-1 non-B subtypes. J. Antimicrob. Chemother. 68, 1994-2002

Apetrei, C., Loussert-Ajaka, I., Collin, G., Letourneur, F., Duca, M., Saragosti, S., Simon, F., Brun-Vezinet, F., 1997. HIV type 1 subtype F sequences in Romanian children and adults. AIDS Res. Hum. Retroviruses 13, 363-365

Apetrei, C., Necula, A., Holm-Hansen, C., Loussert-Ajaka, I., Pandrea, I., Cozmei, C., Streinu-Cercel, A., Pascu, F.R., Negut, E., Molnar, G., Duca, M., Pecec, M., Brun-Vezinet, F., Simon, F., 1998. HIV-1 diversity in Romania. Aids 12, 1079-1085

Bandea, C.I., Ramos, A., Pieniazek, D., Pascu, R., Tanuri, A., Schochetman, G., Rayfield, M.A., 1995. Epidemiologic and evolutionary relationships between Romanian and Brazilian HIV-subtype F strains. Emerg. Infect. Dis. 1, 91-93 
Burke, D.S., 1997. Recombination in HIV: an important viral evolutionary strategy. Emerg. Infect. Dis. 3, 253-259

Ciccozzi, M., Santoro, M.M., Giovanetti, M., Andrissi, L., Bertoli, A., Ciotti, M., 2012. HIV-1 non-B subtypes in Italy: a growing trend. New Microbiol. 35, 377-386

Drummond, A.J., Suchard, M.A., Xie, D., Rambaut, A., 2012. Bayesian phylogenetics with BEAUti and the BEAST 1.7. Mol. Biol. Evol. 29, 1969-1973

Gao, F., Bailes, E., Robertson, D.L., Chen, Y., Rodenburg, C.M., Michael, S.F., Cummins, L.B., Arthur, L.O., Peeters, M., Shaw, G.M., Sharp, P.M., Hahn, B.H., 1999. Origin of HIV-1 in the chimpanzee Pan troglodytes troglodytes. Nature 397, 436-441

Hall, B.G., 2013. Building phylogenetic trees from molecular data with MEGA. Mol. Biol. Evol. 30, 12291235

Hemelaar, J., Gouws, E., Ghys, P.D., Osmanov, S.W.-U.N.f.H. Isolation and Characterisation, 2011. Global trends in molecular epidemiology of HIV-1 during 2000-2007. Aids 25, 679-689

Korber, B., Muldoon, M., Theiler, J., Gao, F., Gupta, R., Lapedes, A., Hahn, B.H., Wolinsky, S., Bhattacharya, T., 2000. Timing the ancestor of the HIV-1 pandemic strains. Science 288, 1789-1796

Leitner, T., Escanilla, D., Franzen, C., Uhlen, M., Albert, J., 1996. Accurate reconstruction of a known HIV-1 transmission history by phylogenetic tree analysis. Proc. Natl. Acad. Sci. U.S.A. 93, 10864-10869

Lewis, F., Hughes, G.J., Rambaut, A., Pozniak, A., Leigh Brown, A.J., 2008. Episodic sexual transmission of HIV revealed by molecular phylodynamics. PLoS Med. 5, e50

Louwagie, J., Delwart, E.L., Mullins, J.I., McCutchan, F.E., Eddy, G., Burke, D.S., 1994. Genetic analysis of HIV-1 isolates from Brazil reveals presence of two distinct genetic subtypes. AIDS Res. Hum. Retroviruses 10, 561-567

Mbisa, J.L., Hue, S., Buckton, A.J., Myers, R.E., Duiculescu, D., Ene, L., Oprea, C., Tardei, G., Rugina, S., Mardarescu, M., Floch, C., Notheis, G., Zohrer, B., Cane, P.A., Pillay, D., 2012. Phylodynamic and phylogeographic patterns of the HIV type 1 subtype F1 parenteral epidemic in Romania. AIDS Res. Hum. Retroviruses 28, 1161-1166

McConnell, M.J., Docobo-Perez, F., Mata, R.C., Fernandez-Cuenca, F., Viciana, P., Lopez-Cortes, L.F., Trastoy, M., Pachon, J., Perez-Romero, P., 2008. Molecular epidemiology of HIV type 1 in newly diagnosed patients in southern Spain. AIDS Res. Hum. Retroviruses 24, 881-887

Mehta, S.R., Wertheim, J.O., Delport, W., Ene, L., Tardei, G., Duiculescu, D., Pond, S.L., Smith, D.M., 2011 Using phylogeography to characterize the origins of the HIV-1 subtype F epidemic in Romania. Infect. Genet. Evol. 11, 975-979

Miri, L., Wakrim, L., Kassar, H., Hemminki, K., Khyatti, M., 2014. Impact of Immigration on HIV-1 molecular epidemiology in West Africa, Maghreb and Southern Europe. AIDS Rev. 16, 109-116

Paraskevis, D., Nikolopoulos, G., Fotiou, A., Tsiara, C., Paraskeva, D., Sypsa, V., Lazanas, M., Gargalianos, P., Psichogiou, M., Skoutelis, A., Wiessing, L., Friedman, S.R., Jarlais, D.C., Terzidou, M., Kremastinou, J., Malliori, M., Hatzakis, A., 2013. Economic recession and emergence of an HIV-1 outbreak among drug injectors in Athens metropolitan area: a longitudinal study. PLoS ONE 8, e78941

Paraskevis, D., Pybus, O., Magiorkinis, G., Hatzakis, A., Wensing, A.M., van de Vijver, D.A., Albert, J., Angarano, G., Asjo, B., Balotta, C., Boeri, E., Camacho, R., Chaix, M.L., Coughlan, S., Costagliola, D., De Luca, A., de Mendoza, C., Derdelinckx, I., Grossman, Z., Hamouda, O., Hoepelman, I., Horban, A., Korn, K., Kucherer, C., Leitner, T., Loveday, C., Macrae, E., Maljkovic-Berry, I., Meyer, L., Nielsen, C., Op de Coul, E.L., Ormaasen, V., Perrin, L., Puchhammer-Stockl, E., Ruiz, L., Salminen, M.O., Schmit, J.C., Schuurman, R., Soriano, V., Stanczak, J., Stanojevic, M., Struck, D., Van Laethem, K., Violin, M., Yerly, S., Zazzi, M., Boucher, C.A., Vandamme, A.M., 2009. Tracing the HIV-1 subtype B mobility in Europe: a phylogeographic approach. Retrovirology 6, 49

Peeters, M., Honore, C., Huet, T., Bedjabaga, L., Ossari, S., Bussi, P., Cooper, R.W., Delaporte, E., 1989. Isolation and partial characterization of an HIV-related virus occurring naturally in chimpanzees in Gabon. AIDS 3, 625-630

Pernas, B., Grandal, M., Mena, A., Castro-Iglesias, A., Canizares, A., Wyles, D.L., Lopez-Calvo, S., Pertega, S., Rodriguez-Osorio, I., Pedreira, J.D., Poveda, E., 2014. High prevalence of subtype F in newly diagnosed HIV-1 persons in Northwest Spain and evidence for impaired treatment response. AIDS 28 , $1837-1840$

Plantier, J.C., Leoz, M., Dickerson, J.E., De Oliveira, F., Cordonnier, F., Lemee, V., Damond, F., Robertson, D.L., Simon, F., 2009. A new human immunodeficiency virus derived from gorillas. Nat. Med. 15, 871872

Price, M.N., Dehal, P.S., Arkin, A.P., 2010. FastTree 2 - approximately maximum-likelihood trees for large alignments. PLoS ONE 5, e9490

Rambaut, A., Posada, D., Crandall, K.A., Holmes, E.C., 2004. The causes and consequences of HIV evolution. Nat. Rev. Genet. 5, 52-61 
Stanojevic, M., Alexiev, I., Beshkov, D., Gokengin, D., Mezei, M., Minarovits, J., Otelea, D., Paraschiv, S., Poljak, M., Zidovec-Lepej, S., Paraskevis, D., 2012. HIV1 molecular epidemiology in the Balkans: a melting pot for high genetic diversity. AIDS Rev. 14, 28-36

Struck, D., Lawyer, G., Ternes, A.M., Schmit, J.C., Bercoff, D.P., 2015. COMET: adaptive context-based modeling for ultrafast HIV-1 subtype identification. Nucleic Acids Res. 42, e144

Thomson, M.M., Fernandez-Garcia, A., Delgado, E., Vega, Y., Diez-Fuertes, F., Sanchez-Martinez, M., Pinilla, M., Castro, M.A., Marino, A., Ordonez, P., Ocampo, A., da Silva, A.R., Perez-Castro, S., LopezAlvarez, M.J., Trigo, M., Perez-Alvarez, L., 2012. Rapid expansion of a HIV-1 subtype F cluster of recent origin among men who have sex with men in Galicia, Spain. J. Acquir. Immune Defic. Syndr. 59, e49-51

UNAIDS, 2013. Global report: UNAIDS Report on the global AIDS epidemic 2013. Van Heuverswyn, F., Li, Y., Neel, C., Bailes, E., Keele, B.F., Liu, W., Loul, S., Butel, C., Liegeois, F., Bienvenue, Y., Ngolle, E.M., Sharp, P.M., Shaw, G.M., Delaporte, E., Hahn, B.H., Peeters, M., 2006. Human immunodeficiency viruses: SIV infection in wild gorillas. Nature 444, 164

Volz, E.M., Kosakovsky Pond, S.L., Ward, M.J., Leigh Brown, A.J., Frost, S.D., 2009. Phylodynamics of infectious disease epidemics. Genetics 183, 1421-1430

Von Wyl, V., Kouyos, R.D., Yerly, S., Boni, J., Shah, C., Burgisser, P., Klimkait, T., Weber, R., Hirschel, B., Cavassini, M., Staehelin, C., Battegay, M., Vernazza, P.L., Bernasconi, E., Ledergerber, B., Bonhoeffer, S., Gunthard, H.F., Swiss, H.I.V.C.S., 2011. The role of migration and domestic transmission in the spread of HIV-1 non-B subtypes in Switzerland. J. Infect. Dis. 204, 1095-1103

Yebra, G., de Mulder, M., Martin, L., Rodriguez, C., Labarga, P., Viciana, I., Berenguer, J., Aleman, M.R., Pineda, J.A., Garcia, F., Holguin, A.A.R.N. Cohort of the Spanish, 2012. Most HIV type 1 non-B infections in the Spanish cohort of antiretroviral treatment-naive HIV-infected patients (CoRIS) are due to recombinant viruses. J. Clin. Microbiol. 50, 407-413 